\title{
Editorial
}

\section{Autosis: a new addition to the cell death tower of babel}

\author{
C Muñoz-Pinedo, ${ }^{*, 1}$ and SJ Martin ${ }^{2,3}$ \\ Cell Death and Disease (2014) 5, e1319; doi:10.1038/cddis.2014.246; published online 10 July 2014
}

Autophagy is a cellular recycling and stress response that degrades organelles and long-lived proteins and serves to protect cells from the potential damage induced by dysfunctional organelles and protein aggregates. ${ }^{1}$ Autophagy can also be used as a recycling or salvage process to provide amino acids, nucleotides and other building blocks to protect cells from some, but not all, forms of starvation. ${ }^{2,3}$

Given that autophagy is part of the cellular stress response, it is perhaps not surprising that this process can also influence cell death decisions. Similar to the endoplasmic reticulum stress response, autophagy is predominantly cytoprotective, but excessive or persistent autophagy can also be cytotoxic. ${ }^{4}$ Similarly, the p53-dependent stress response, which is primarily concerned with coordinating DNA damage-associated repair, can result in apoptosis above a certain threshold of p53 activation. ${ }^{5}$ This makes good biological sense, as cells with excessive DNA damage are risky to repair due to the potential for the development of cancer. Indeed, many biological responses are governed by threshold effects, above and below which either no response occurs or a qualitatively different response is initiated. Other examples include activation of Myc, Ras or B-Raf, which are normally drivers of cell proliferation, but can promote the diametrically opposite outcomes of apoptosis, replicative senescence or autophagic cell death when deregulated. ${ }^{6-8}$

Thus, it is not particularly surprising that excessive autophagy can promote cell death rather than cell survival in several situations. ${ }^{4}$ To distinguish cell death accompanied by signs of autophagy (i.e. 'cell death with autophagy') from cell death as a consequence of autophagy, the term 'autophagic cell death' is employed to define instances in which the autophagy machinery is required for cell death. ${ }^{9,10}$ Autophagic cell death has been reported during Drosophila development ${ }^{11,12}$ in response to ischemia ${ }^{13,14}$ as a consequence of deregulated Ras activation, ${ }^{15}$ upon inhibition of caspase-10 in multiple myeloma, ${ }^{16}$ or upon treatment of apoptosis-deficient cells with chemotherapeutic drugs. ${ }^{17}$

Levine and colleagues have recently characterized a form of autophagic cell death induced by a synthetic autophagyinducing peptide, comprising the viral Tat peptide fused to a small peptide sequence from Beclin-1. ${ }^{18}$ It is unclear why the Tat-Beclin-1 peptide promotes autophagy, or indeed autophagic cell death, or whether this mimics a bona fide physiological stress. Nonetheless, Liu et al. have characterized the morphological features of cell death induced by Tat-Beclin 1, a form of cell death that can be partially rescued by knockdown of Atg13 or Atg14, as well as 3-methyladenine. They confirmed that cell death in this context was not apoptotic or necroptotic and also observed the vacuolated cytoplasm characteristic of recent descriptions of autophagic cell death. ${ }^{15}$ Moreover, a small minority $(\sim 1 \%)$ of cells dying as a consequence of amino acid/serum starvation presented the same morphological features as cells treated with Tat-Beclin-1 peptide. Some of these descriptive morphological features were also seen in a model of in vivo permanent brain ischemia, in which neurons appear to die with features of autophagic cell death.

A compound library screen revealed that the $\mathrm{Na}^{+}, \mathrm{K}^{+}$ATPase, an ionic pump that consumes $20 \%$ of cellular ATP (and up to $66 \%$ in neurons), participates in autophagic cell death in this setting. Consistent with this, cardiac glycosideschemical inhibitors of the $\mathrm{Na}^{+}, \mathrm{K}^{+}$-ATPase-prevented autophagic cell death induced by amino acid/serum starvation. Liu et al. also showed that, as reported previously in a similar model, a cardiac glycoside (neriifolin) dramatically reduced brain damage in neonatal rats subjected to brain ischemia. $^{19}$

Levine and colleagues have suggested that the term 'Autosis' be used to define the form of autophagic cell death characterized in their study. However, it is not clear that the introduction of a new name for this form of cell death is really warranted. The primary case made by the authors for the introduction of the new term (autosis) is that the term 'autophagic cell death' has become controversial and has sometimes been confused with cells dying with features of autophagy. This is as distinct from cells dying as a consequence of excessive autophagy (i.e. autophagic cell death). The introduction of yet another new term for a cell death modality may not be that helpful for a number of reasons.

First, the distinction between autophagic cell death and cell death accompanied by features of autophagy is now well recognized. Second, it is too early to say whether the mode of cell death reported by Levine and colleagues represents a physiological mode of cell death, as this has been studied almost entirely in the context of an artificial Tat-Beclin fusion peptide. The evidence that this mode of cell death occurs in vivo is very preliminary and entirely descriptive. Third, when

${ }^{1}$ Cell Death Regulation Group, IDIBELL (Institut d'Investigació Biomèdica de Bellvitge) - Hospital Duran i Reynals $3^{\text {a }}$ planta, Gran Via de L'Hospitalet 199, L'Hospitalet, Barcelona 08908, Spain; ${ }^{2}$ Molecular Cell Biology Laboratory, Department of Genetics, Trinity College, Dublin 2, Ireland and ${ }^{3}$ Cellular Biotechnology Laboratory, Saint-Petersburg State Institute of Technology, Moskovskii prospekt, Saint Petersburg, Russia

${ }^{*}$ Corresponding author: C Muñoz-Pinedo, Cell Death Regulation Group, IDIBELL (Institut d'Investigació Biomèdica de Bellvitge) - Hospital Duran i Reynals $3^{\text {a }}$ planta, Gran Via de L'Hospitalet 199, L'Hospitalet, Barcelona 08908, Spain. Tel: +34 93260 7130; Fax: +34 93260 7426; E-mail: cmunoz@idibell.cat 
re-naming a process, as in this situation, there should be a strong rationale for doing so. To do otherwise is to risk a proliferation of new terms for what is essentially the same process. Indeed, this has already happened to some degree in the cell death field. Thus, we have apoptosis, necrosis, anoikis, pyroptosis, aponecrosis, necroptosis, programmed necrosis, autophagic cell death, entosis and countless more names for cell death triggered under different conditions or displaying a distinct morphology. Some forms of cell death have even been named after compounds or stimuli that trigger them (e.g. Ferroptosis, Anoikis, mitotic catastrophe). Here, it is proposed that autophagic cell deaths exhibiting certain features (i.e. mediated by autophagy genes and characterized by focal perinuclear swelling) should be named Autosis. However, the logic for introducing a new name for what is, in essence, simply autophagic cell death is not particularly compelling. For example, staurosporine is a well-known inducer of apoptosis, but because of its properties as a kinase inhibitor it induces an atypical apoptosis with ballooning of cells and some features reminiscent of the early stages of necrosis. Should this be called 'Stauroptosis' because of its distinct morphological features?

At present, there does not appear to be a strong case for the introduction of a new term to describe another example of autophagic cell death. Perhaps if subsequent studies determine that this subroutine of autophagic cell death is indeed mechanistically distinct and is important under physiological conditions, this might be appropriate. We would note that the Cell Death Nomenclature committee recommends that the term Autophagic Cell Death should be used to refer to instances of cell death that 'can be suppressed by the inhibition of the autophagic pathway by chemicals (e.g. agents that target VPS34) and/or genetic means' and are not mediated by caspases or necroptotic mediators. Coining a new term for a subtype of cell death that clearly fits into an existing category is unwarranted and contributes to creating a scientific tower of Babel where it becomes progressively difficult to know what we are all talking about.

Notwithstanding these quibbles about the introduction of a new term for autophagic cell death, it is possible that the inhibitors of this process described by Levine and colleagues may have therapeutic implications in conditions where death by autophagy may be contributory to the underlying pathology.

\section{Conflict of Interest}

The authors declare no conflict of interest.

Acknowledgements. Javier Galindo is acknowledged for his presentations in journal clubs. CM-P's lab is funded by Fundació Marató TV3 (Grant 111630/31) and Fondo de Investigaciones Sanitarias of Spain (PI13/00139). SJM is supported by grants from Science Foundation Ireland as well as a grant from the Russian government for state support of scientific research.

1. Mizushima N, Komatsu M. Cell 2011; 147: 728-741.

2. Boya $P$ et al. Mol Cell Biol 2005; 25: 1025-1040.

3. Ramirez-Peinado S et al. J Biol Chem 2013; 288: 30387-30398.

4. Martin SJ. Autophagy 2011; 7: 922-923.

5. Lowe SW et al. Cell 1993; 74: 957-967.

6. Evan Gl et al. Cell 1992; 69: 119-128.

7. Serrano M et al. Cell 1997; 88: 593-602.

8. Michaloglou C et al. Nature 2005; 436: 720-724.

9. Marino G et al. Nat Rev Mol Cell Biol 2014; 15: 81-94.

10. Galluzzi L et al. Cell Death Differ 2012; 19: 107-120.

11. Berry DL, Baehrecke EH. Cell 2007; 131: 1137-1148.

12. Denton D et al. Curr Biol 2009; 19: 1741-1746.

13. Zhang $X$ et al. Autophagy 2013; 9: 1321-1333.

14. Puyal J et al. Ann Neurol 2009; 66: 378-389.

15. Elgendy $\mathrm{M}$ et al. Mol Cell 2011; 42: 23-35.

16. Lamy L et al. Cancer Cell 2013; 23: 435-449.

17. Shimizu $S$ et al. Nat Cell Biol 2004; 6: 1221-1228.

18. Liu $Y$ et al. Proc Natl Acad Sci USA 2013; 110: 20364-20371.

19. Wang JK et al. Proc Natl Acad Sci USA 2006; 103: 10461-10466.

(i) (8) $\odot$ Cell Death and Disease is an open-access journal published by Nature Publishing Group. This work is licensed under a Creative Commons Attribution-NonCommercialNoDerivs 3.0 Unported License. The images or other third party material in this article are included in the article's Creative Commons license, unless indicated otherwise in the credit line; if the material is not included under the Creative Commons license, users will need to obtain permission from the license holder to reproduce the material. To view a copy of this license, visit http://creativecommons.org/ licenses/by-nc-nd/3.0/ 